\title{
Research on Configuration Optimization of ACIDC Charging Equipment under Large Scale Access of Electric Vehicles Considering Disordered Charging
}

\author{
Lingyi $\mathrm{Li}^{1}$ and Tie Chen ${ }^{2, *}$ \\ ${ }^{1}$ College of Electrical Engineering \& New Energy, China Three Gorges University, Yichang, 443002, \\ China \\ ${ }^{2}$ College of Electrical Engineering \& New Energy, China Three Gorges University, Yichang, 443002, \\ China \\ ${ }^{*}$ Corresponding author
}

\begin{abstract}
Keywords: Electric vehicle, Charging law, Monte Carlo simulation, AC/DC charging equipment, Configuration optimization.
\end{abstract}

\begin{abstract}
In order to meet the demand of large-scale charging of electric vehicles effectively, it is urgent to carry out research on configuration optimization of $\mathrm{AC} / \mathrm{DC}$ charging equipment. For this reason, the charging law is modeled on the basis of the charging characteristic data of a small-scale electric vehicle in this paper, with which the charging behavior of the large-scale electric vehicles is deduced by adopting Monte Carlo simulation method. Then, some model assumptions are given and the equivalent load curve is acquired. Furtherly, the optimization objective function of AC/DC charging equipment configuration for minimizing the equipment investment and the peak-to-valley difference of disordered charging load is constructed. Finally, the optimal solutions, i.e., referential allocation quantities of various charging equipment are obtained by solving the optimization problem with the related constraints, which can provide certain theoretical basis for the configuration planning of $\mathrm{AC} / \mathrm{DC}$ charging equipment under connection of large-scale electric vehicles.
\end{abstract}

\section{Introduction}

In order to reduce the dependence on petroleum resources and solve the world energy security problems, governments in various countries are vigorously developing the green and environmental protection electric vehicle industry under the background of the development and utilization of new energy resources ${ }^{[1]}$. The relevant issues have also attracted widespread attention.

The planning and construction of charging infrastructure is directly related to the promotion effect and scale of electric vehicles. The charging behavior of electric vehicles has great uncertainty and randomness in time and space ${ }^{[2]}$. When large-scale electric vehicles are connected to the power grid in disorder, their charging and discharging behavior will affect many aspects of the power grid, such as power system scheduling, distribution network peak and valley difference, power quality, etc. When the situation is serious, it may cause great impact on the operation of distribution network $^{[3]}$. Therefore, it is necessary to optimize the optimal configuration of electric vehicle charging equipment in combination with the variation of the characteristic quantity of user charging behavior.

The configuration of electric vehicle charging equipment is a multi-objective and multi-variable decision-making optimization problem. Many scholars have conducted related research on these problems. Literature [3] analyzed many factors affecting the choice of fast and slow charging modes. Based on the probability model, the calculation method and test index of the proportionality of fast and slow charging facilities were proposed. Literature [4], based on the predicted data of electric vehicle ownership in hanzhong city at the end of 2020, simulated and predicted the overall charging load curve under corresponding modes. Literature [5] considered different types of charging demands, established an integer linear programming model aiming at the minimum of total investment cost, operation and maintenance cost, power cost and user cost of charging station The 
above research has achieved some research results around charging equipment configuration, but has not yet made a comprehensive analysis of the impact of peak-valley difference and charging equipment investment under the large-scale access of electric vehicles. This paper analyzes the charging characteristics of small-scale electric vehicles, constructs an AC/DC charging device configuration optimization objective function that minimizes equipment investment and disordered charging load peak-to-valley difference, and finally optimizes the solution by combining relevant constraint analysis.

\section{Modeling of Charging Law of Electric Vehicle}

Common probability distribution forms are normal distribution, $\Gamma$ distribution, Rayleigh distribution, etc., which correspond to different probability density functions. The probability density functions of normal distribution and $\Gamma$ distribution are as follows:

(1) Normal distribution:

$$
f(\chi, \mu, \sigma)=\frac{1}{\sigma \sqrt{2 \pi}} e^{-\frac{(\chi-\mu)^{2}}{2 \sigma^{2}}} .
$$

Where $\mu$ is the mathematical expectation and $\sigma$ is the standard deviation.

(2) $\Gamma$ distribution:

$$
\begin{gathered}
f(\chi, \beta, \alpha)=\frac{\beta^{\alpha}}{\Gamma(\alpha)} \chi^{\alpha-1} e^{-\beta \chi}, \chi>0 \\
\Gamma(\alpha)=\int_{0}^{+\infty} t^{\alpha-1} e^{-t} d t .
\end{gathered}
$$

Where $\alpha$ is the shape parameter and $\beta$ is the scale parameter.

The charging behavior characteristics of electric vehicles mainly include the beginning time for charging, charging connection duration, electric quantity of charging, etc., which satisfy a certain probability distribution form. The charging characteristic data of 100 electric vehicles in a city on a certain day are selected for distribution analysis. The charging records of 10 vehicles are shown in Table 1.

Table 1. Electric vehicle charging records

\begin{tabular}{cccc}
\hline Number & $\begin{array}{c}\text { The start of } \\
\text { charging }\end{array}$ & $\begin{array}{c}\text { Time of Charging } \\
\text { Connection } / \mathrm{h}\end{array}$ & $\begin{array}{c}\text { Charging } \\
\text { power } / \mathrm{kWh}\end{array}$ \\
\hline EV 1 & $8: 03$ & 7.93 & 14.83 \\
EV 2 & $17: 13$ & 0.80 & 3.24 \\
EV 3 & $7: 05$ & 10.52 & 27.36 \\
EV 4 & $11: 09$ & 4.70 & 7.64 \\
EV 5 & $17: 26$ & 1.06 & 1.41 \\
EV 6 & $12: 08$ & 3.47 & 22.78 \\
EV 7 & $18: 22$ & 4.77 & 6.76 \\
EV 8 & $12: 34$ & 3.70 & 3.43 \\
EV 9 & $8: 34$ & 7.35 & 15.66 \\
EV 10 & $9: 18$ & 3.54 & 17.72 \\
\hline
\end{tabular}

Distribution analysis of access time of 100 electric vehicles shows that it satisfies normal distribution. The mathematical expectation of probability density function $\mu$ is 0.5764 (normalized value, 24 hours corresponding to 1), and the standard deviation $\sigma$ is 0.21 .

Distribution analysis of the charging connection time of 100 electric vehicles shows that it satisfies $\Gamma$ distribution. The shape parameter of the probability density function $\alpha$ is 1.7814 , and the scale parameter $\beta$ is 7.4824. And distribution analysis of the charging electric quantity of 100 
electric vehicles shows that it also satisfies $\Gamma$ distribution. The shape parameter of the probability density function $\alpha$ is 2.3480 , and the scale parameter $\beta$ is 7.0055 .

\section{Analysis of Large-scale Charging Behavior}

Monte Carlo method, also known as statistical simulation method, is a stochastic computing simulation method based on probability and statistical theory ${ }^{[6]}$. The research scale is set to 10,000 vehicles. Since the subsequent optimization analysis uses the equivalent peak-to-valley difference, that is, the number of connected electric vehicles, so the scale of the charging start time and the charging connection time is mainly analyzed here.

Expanding the given 100 charging samples to 10,000 based on Monte Carlo simulation method. The distribution of 10,000 electric vehicles with different charging start time and different charging connection time are shown in Figure 1 and Figure 2 respectively.

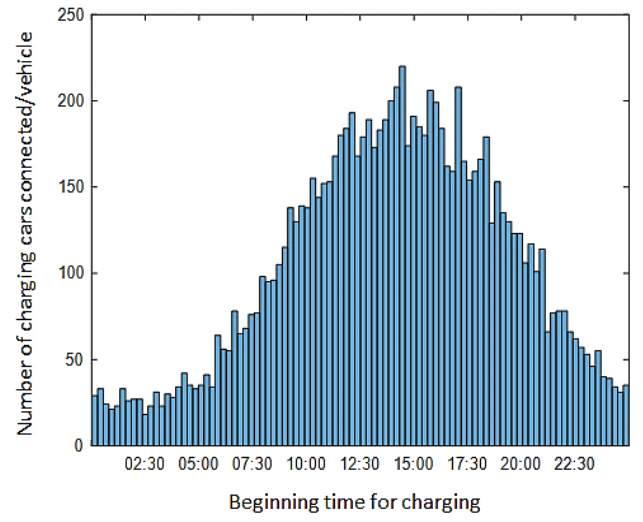

Figure 1. Distribution of ten thousand electric cars with different beginning time for charging

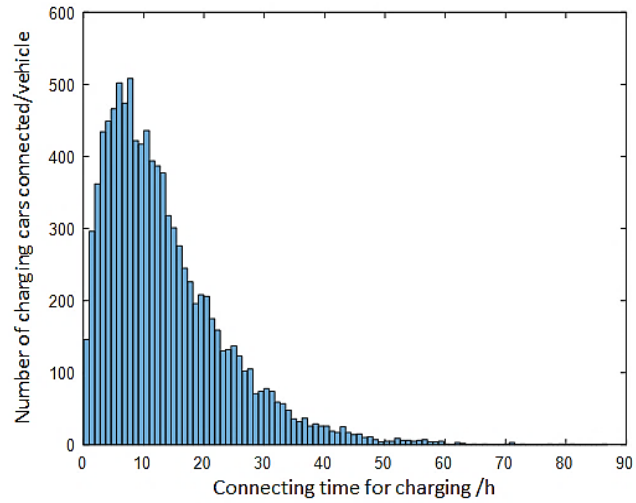

Figure 2. Distribution of ten thousand electric cars with different connecting time for charging

Considering that there is no necessary relationship between the charging connection time, charging electric quantity and charging start time of each electric vehicle, the charging connection time and charging electric quantity corresponding to each charging start time are determined by random method. Finally, 10,000 large-scale electric vehicle data were obtained.

\section{Charging Equivalent Load of Large-scale Electric Vehicle}

\section{Model Assumptions}

Considering the random charging behavior of each vehicle, in order to simplify the calculation, the number of electric vehicles connected at each time is regarded as the equivalent load. The following assumptions are made:

(1)Each electric car is only charged once a day.

(2)When the electric vehicle charging interface is connected, it is charged. 
(3)At the end of the day, if an electric car is still charging, consider it as the effect of the previous day on the start time.

(4)The connection of electric vehicles is judged according to the time interval. With $15 \mathrm{~min}$ as the interval, the total duration is $24 \mathrm{~h}$, and the interval ordinal is $1,2, \ldots, 96$ in turn. Connecting at any time in an interval is considered to be connected at that time. Disconnection at any time in an interval period will be regarded as disconnection in the next period of that period.

\section{Equivalent Load Curve}

Based on the charging start time and the charging connection duration, the end time is determined first, and the interval corresponding to the start time and the end time is determined according to the assumption (4). The equivalent load curve is shown in Figure 3.

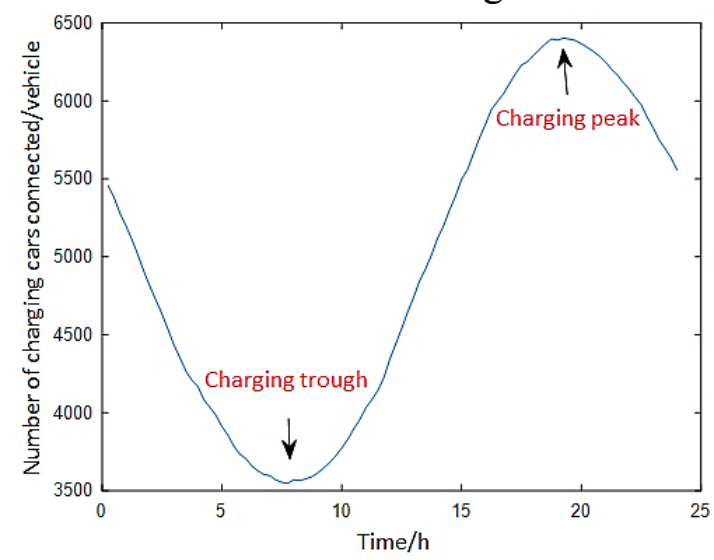

Figure 3. Equivalent load curve of charging electric cars

\section{Optimization of AC/DC Charging Equipment Configuration}

\section{Objective Function}

Information about power levels for different charging equipment is shown in Table 2.

Table 2. Electric vehicle charging power levels

\begin{tabular}{cccc}
\hline & AC level 1 & AC level 2 & DC \\
\hline power(kW) & $1.5-3$ & $10-25$ & $40-100$ \\
Cost per device(Ten thousand yuan) & 0.5 & 1.8 & 40 \\
\hline
\end{tabular}

Let $\mathrm{N} 1, \mathrm{~N} 2$, and $\mathrm{N} 3$ be AC level 1, AC level 2, and DC charging equipment number, then the equipment investment function $f_{1}$ can be expressed as:

$$
f_{1}=0.5 N_{1}+1.8 N_{2}+40 N_{3} \text {. }
$$

In order to make the charge peak-to-valley difference as small as possible, the minimum power charging is used at the peak, and the maximum power charging is used in the low valley. Let F1, F2 and $\mathrm{F} 3$ respectively be the number of $\mathrm{AC}$ level 1, AC level 2 and DC charging devices accessed at peak charging load, and G1, G2 and G3 are the number of AC level 1, AC level 2 and DC charging devices accessed at low charging load, then the peak-valley difference function $f_{2}$ can be expressed as follows:

$$
f_{2}=1.5 F_{1}+10 F_{2}+40 F_{3}-3 G_{1}-25 G_{2}-100 G_{3} .
$$

To facilitate quantitative analysis, the comprehensive objective function is defined as follows:

$$
\min f=10 f_{1}+f_{2} .
$$

\section{Constraints}

In order to find the optimal solution, the constraints are as follows: 


$$
\left\{\begin{array}{l}
N_{1}+N_{2}+N_{3}=10000 \\
F_{1}+F_{2}+F_{3}=N F_{\max } \\
G_{1}+G_{2}+G_{3}=N G_{\max } \\
N_{2}+N_{3} \geq N_{3.0} \\
N_{3} \geq N_{25.0} \\
0 \leq F_{i} \leq N_{i}(i=1,2,3) \\
0 \leq G_{i} \leq N_{i}(i=1,2,3)
\end{array}\right.
$$

Where $N F_{\text {max }}$ and $N G_{\text {max }}$ are the number of electric vehicles connected in peak and trough periods of charging load, $N_{3.0}$ is the number of electric vehicles with charging power greater than $3.0 \mathrm{~kW}$, and $N_{25.0}$ is the number of electric vehicles with charging power greater than $25.0 \mathrm{~kW}$.

\section{Solution of the Model}

The objective function and constraints are shown above. It can be seen from the above formula that the multi-objective optimization problem can be transformed into a linear programming problem. The solution can be obtained:

$$
N_{1}=6403, N_{2}=3538, N_{3}=59
$$

When the number of AC 1, AC 2 and DC charging equipment accessed is 6403, 3538 and 59 respectively, the optimal solution of the multi-objective function defined can be obtained. Based on the Monte Carlo simulation idea, the above optimization solution process is repeated 100 times. After averaging and combining the total results, the final configuration results can be obtained.

\section{Conclusion}

Based on the characteristics data of small-scale electric vehicle charging behavior, this paper puts forward a method for configuring the AC/DC charging equipment under large-scale access of electric vehicles considering unordered charging. It can provide some theoretical basis for the configuration planning of $\mathrm{AC} / \mathrm{DC}$ charging equipment under large-scale access of electric vehicles.

\section{References}

[1] Xiao Xiangning, Wen Jianfeng, Tao Shun, et al. Study and recommendations of the key issues in planning of electric vehicles' charging facilities[J]. Transactions of China Electrotechnical Society, 2014, 29(8):1-10(in Chinese).

[2] Li Huiling, Bai Xiaomin. Impacts of electric vehicles charging on distribution grid[J]. Automation of Electric Power Systems, 2011, 35(17): 38-43(in Chinese).

[3] Tao Shun, Xiao Xiangning, Wen Jianfeng, et al. Configuration ratio for distributed electrical vehicle charging infrastructures[J]. Transactions of China Electrotechnical Society, 2014, 29(8): 11-19(in Chinese).

[4] Tang Kaiyu, Yin Shaoqi. Electric vehicle charging load forecasting and configuration ratio analysis under slow and fast charging mode[J]. Shaanxi Power, 2017, 45(8): 106-110(in Chinese).

[5] Jia Long, Hu Zechun, Song Yonghua. An integrated planning of electric vehicle charging facilities for urban area considering different types of charging demands[J]. Power System Technology, 2016, 40(9): 2579-2587(in Chinese).

[6] Wang Y, Infield D. Markov Chain Monte Carlo simulation of electric vehicle use for network integration studies[J]. International Journal of Electrical Power \& Energy Systems, 2018, 99:85-94. 